\title{
Interval and fuzzy dynamic analysis of finite element models with superelements
}

\author{
Hilde De Gersem*, David Moens, Wim Desmet, Dirk Vandepitte \\ Katholieke Universiteit Leuven \\ Department of Mechanical Engineering, PMA \\ Celestijnenlaan 300B, B-3001 Heverlee, Belgium
}

\begin{abstract}
This paper uses the interval and fuzzy finite element method for the eigenvalue and frequency response function analysis of structures with uncertain parameters. In order to reduce the calculation time of the interval and fuzzy analyses, these non-probabilistic methods are combined with the component mode synthesis technique. Special attention is paid to the effect of uncertainties on the mathematical description of this substructuring technique. All concepts are illustrated through a benchmark structure example.
\end{abstract}

Key words: uncertainty, interval finite element method, fuzzy finite element method, eigenvalues, frequency response function, component mode synthesis, substructuring

\section{Introduction}

During the last decades, the exponential growth of computational power has enabled the finite element (FE) analysis of very complex and detailed numerical models of new designs and structures. However, it is often very difficult to define a reliable finite element model for numerical simulation and validation, as some numerical properties may be uncertain, e.g. due to manufacturing tolerances, variability on material characteristics, modelling simplications etc. Especially during the design of a product, numerical descriptions of model details are often not available or only partially known. Due to the ever growing demands imposed on new products, it is of utmost importance for design engineers to investigate the influence of all relevant uncertainties on the static and/or dynamic behaviour of structures. Hence, non-deterministic approaches are gaining interest in design and development areas. Nowadays, these techniques are applied in enhanced numerical tools as reliability assessment, robustness analysis and design optimisation.

The probabilistic concept is already well established for the extension of the deterministic finite element method towards uncertainty assessment. This has led to a number of probabilistic finite element procedures [1], that are often used in conjunction with Monte Carlo Simulations. Probabilistic methods are especially suitable in case of model variabilities for which information on both the range and the probability density function is available. However, the use of a probabilistic approach can lead to subjective results if the amount of statistical data on the uncertainties is limited and approximations and subjective information need to be included [2].

The fuzzy set theory, introduced by Zadeh [3] in 1965, provides a concept for the description of linguistic or subjective knowledge and incomplete data in a non-probabilistic manner. The use of Zadeh's concept in the finite element context has led to the development of the fuzzy finite element method [4]. Its aim is to calculate the membership function of an output quantity, based on the fuzzy description of the uncertain input parameters. By using the $\alpha$-level technique, the interval finite element method (IFEM) forms the core of the fuzzy procedure.

Over the last decade, several interval FE procedures have been reported, such as the vertex method, the global optimisation approach and the interval arithmetic approach. A hybrid procedure for the interval frequency response function (FRF) calculation has been developed and

\footnotetext{
*Corresponding author. Tel.: +32 16 328606; Fax: +32 16 322987; E-mail: hilde.degersem@mech.kuleuven.be
} 
implemented by Moens $[5,6]$. In this approach, a global optimisation step determines the correct intervals on the modal parameters, which are then combined to calculate the FRF upper and lower bounds with an interval arithmetic procedure. Special attention is paid to the limitation of the conservatism and the computational efficiency of the method. The hybrid FRF procedure was first developed for undamped and proportionally damped structures [7], and it is recently extended for fuzzy modal damping parameters [8]. Various numerical case studies prove that the interval and fuzzy finite element method provide useful tools to perform early design validation and optimisation [9].

For industrial size models with a large number of uncertainties, the computation time of the interval and the fuzzy finite element method can be considerable. A reduction in calculation time can be achieved by the substructuring of large models into superelements, which are then independently processed and reduced [10]. Subsequently all reduced superelements are recombined to form the reduced structural system for which the desired output quantities are calculated. However, the introduction of uncertainties in a substructuring technique requires a concept for the description of uncertainty on the superelement level. In this paper, the fuzzy finite element method is combined with the Craig-Bampton component mode synthesis method, in which the static and dynamic behaviour of each superelement are represented by a set of component modes.

This paper describes the basic properties of the fuzzy finite element method in section 2 . This section also gives an overview of the hybrid procedure for fuzzy frequency response function analysis, as developed by Moens. Section 3 describes the basic principles of the component mode synthesis technique. In section 4 , the above mentioned methods are combined for the interval and fuzzy eigenfrequency and frequency response function analysis of uncertain FE models with superelements. Different approaches to handle uncertainties in superelements are proposed. In section 5, interval and fuzzy dynamic analyses are performed on two superelement FE models of the Garteur benchmark aircraft. The accuracy and numerical efficiency of the proposed approaches for superelement uncertainty handling are compared.

\section{The Fuzzy Finite Element Method}

\subsection{Fuzzy Sets}

The concept of fuzzy sets, introduced by Zadeh [3] in 1965, has gained an increasing popularity during the last two decades. Its most important property is that it is capable of describing linguistic and therefore incomplete information in a non-probabilistic manner. Whereas a classical set clearly distinguishes between members and non-members, a fuzzy set introduces a degree of membership, represented by the membership function. For a fuzzy set $\tilde{x}$, the membership function $\mu_{\tilde{x}}(x)$ describes the grade of membership to the fuzzy set for each element $x$ in the domain $X$ :

$$
\tilde{x}=\left\{\left(x, \mu_{\tilde{x}}(x)\right) \mid(x \in X)\left(\mu_{\tilde{x}}(x) \in[0,1]\right)\right\}
$$

If $\mu_{\tilde{x}}(x)=1, x$ is definitely a member of the set $\tilde{x}$, whereas if $\mu_{\tilde{x}}(x)=0, x$ is definitely not a member of the set $\tilde{x}$. For all $x$ with $0<\mu_{\tilde{x}}(x)<1$, the membership is not certain. The most frequently applied membership function shapes are the triangular and Gaussian shape.

\section{$2.2 \quad$ Fuzzy Numerical Analysis}

The fuzzy finite element method (FFEM) aims to obtain a fuzzy description of an FE analysis result, starting from the fuzzy descriptions of all non-deterministic FE model parameters. In practice, the description of uncertain parameters and quantities using fuzzy sets can be implemented using the $\alpha$-level strategy. This approach subdivides the membership function range into a number of $\alpha$-levels. The intersection with the membership function of the input uncertainties at each $\alpha$ level results in an interval $x_{i, \alpha}^{I}=\left[\underline{x}_{i}, \bar{x}_{i}\right]_{\alpha}$. With these input intervals of the $\alpha$-sublevel, an interval finite element (IFE) analysis is performed, resulting in an interval for the analysis result at the considered $\alpha$-level. Finally, the fuzzy solution is assembled from the resulting intervals at each sublevel. Figure 1 illustrates this procedure for a function of two triangular fuzzy parameters.

The fuzzy finite element technique consists of the application of the $\alpha$-level strategy on the numerical procedure of the deterministic FE analysis. Through this procedure, the fuzzy FE 


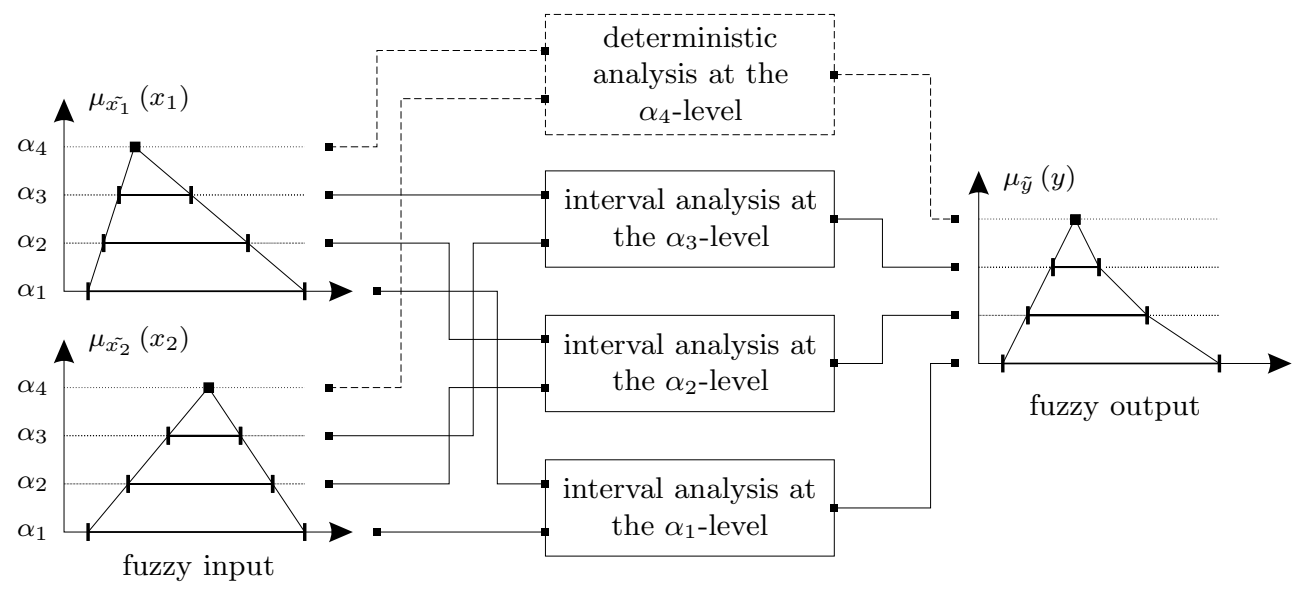

Figure 1: $\alpha$-level strategy with $4 \alpha$-levels, for a function of two triangular fuzzy parameters

analysis can be interpreted as a large-scale sensitivity analysis, which can be used to study the combined effect of the interval bounds of design variables on critical design properties. See [11] for a more comprehensive overview of fuzzy analysis for non-deterministic analysis in engineering design.

Through the $\alpha$-level strategy, the interval problem corresponding to the considered FE analysis forms the core of the fuzzy procedure. A number of general interval solutions for the interval FE problem have been described in literature. The most popular one is the interval arithmetic approach, in which all basic deterministic algebraic operations are replaced by their interval arithmetic counterparts. Although this approach is computationally very inexpensive, it is of little practical use as it tends to largely overestimate the interval outcome of each operation, accumulating in a huge amount of conservatism in the interval analysis result of realistic problems. Therefore, other approaches such as the vertex method [12], the transformation method [13, 14] and the global optimisation method [4] have been proposed for the solution of the underlying interval FE problem. Each of these techniques has its specific disadvantages when applied to realistical engineering problems, such that none of them has been established as standard interval procedure, and the choice of technique depends on the type of the conducted FE analysis. In case of an interval eigenfrequency analysis, the aim is to calculate the minimum and maximum values of the eigenfrequencies of all considered modes, given the input uncertainty intervals. It can easily be shown that a vertex method, which takes all combinations of the boundaries of the input intervals into account, correctly predicts the intervals of output quantities if the problem is monotonic. However, in the application of structural dynamics, it is very hard - if not impossible - to prove the property of monotonicity in a general way. Experience shows that many problems do exhibit a monotonic behaviour, especially when global model parameters are uncertain. For parameters that affect only part of the structure, it is much harder to assess a priori the effect of a parameter change. For those cases, it is necessary to apply a global optimisation procedure in search of the minimum and maximum eigenfrequency values. For the interval frequency response function analysis, a hybrid method has been proposed by Moens, which combines the global optimisation approach and the interval arithmetic approach, resulting in a procedure with an acceptable computational cost and a limited amount of conservatism in the analysis result.

\section{$2.3 \quad$ Fuzzy FRF Analysis}

The fuzzy finite element method for frequency response function analysis combines the concept of fuzzy sets, implemented by the $\alpha$-level strategy, with the modal superposition principle. The first part of the underlying interval procedure consists of an optimisation step, in which the input uncertainty intervals of the FE model are translated to the exact intervals for the modal stiffness and modal mass parameters of the structure, thereby neutralizing all conservatism in the system matrix assembly phase. With the modal stiffness and modal mass intervals, the calculation of the upper and lower FRF bounds is performed by an interval arithmetic procedure. The final interval 
FRFs have been proven to contain only a very limited amount of conservatism. A brief overview of the hybrid method is given below. A complete mathematical description of the method can be found in Moens [5, 6].

Deterministic modal superposition principle. For undamped structures, the deterministic modal superposition principle states that, considering the first $n_{\text {modes }}$ modes, the frequency response function between degrees of freedom $j$ and $k$ equals:

$$
F R F_{j k} \simeq \sum_{i=1}^{n_{\text {modes }}} F R F_{j k}^{i}=\sum_{i=1}^{n_{\text {modes }}} \frac{\phi_{i_{k}} \phi_{i_{j}}}{\left\{\phi_{i}\right\}^{T}[K]\left\{\phi_{i}\right\}-\omega^{2}\left\{\phi_{i}\right\}^{T}[M]\left\{\phi_{i}\right\}}
$$

with $\left\{\phi_{i}\right\}$ the $i^{\text {th }}$ eigenvector of the system and $\phi_{i_{j}}$ the $j^{\text {th }}$ component of the $i^{\text {th }}$ eigenvector. Equation (2) can be reformulated as:

$$
F R F_{j k}=\sum_{i=1}^{n_{\text {modes }}} \frac{1}{\hat{k}_{i}-\omega^{2} \hat{m}_{i}}
$$

with $\hat{k}_{i}$ and $\hat{m}_{i}$ the modal stiffness and modal mass parameters defined as:

$$
\begin{aligned}
\hat{k}_{i} & =\frac{\left\{\phi_{i}\right\}^{T}[K]\left\{\phi_{i}\right\}}{\phi_{i_{j}} \phi_{i_{k}}} \\
\hat{m}_{i} & =\frac{\left\{\phi_{i}\right\}^{T}[M]\left\{\phi_{i}\right\}}{\phi_{i_{j}} \phi_{i_{k}}}
\end{aligned}
$$

The function $\mathcal{D}_{i}(\omega)=\left(\hat{k}_{i}-\omega^{2} \hat{m}_{i}\right)$ expresses the modal response denominator as a function of frequency.

Interval frequency response function analysis. The modal superposition principle has been translated into an interval finite element procedure for FRF analysis. Figure 2 gives a graphical overview of the translation of the deterministic algorithm into an interval procedure for the undamped case. The interval translation shows that the total envelope FRF can be calculated in three successive steps:

1. For all $n_{\text {modes }}$ taken into account, the correct ranges of the modal parameters $\left\langle\hat{k}_{i}\right\rangle$ and $\left\langle\hat{m}_{i}\right\rangle$ are determined using a global minimisation and maximisation over the vector space $\{\mathbf{x}\}$ defined by the input interval parameters:

$$
\begin{aligned}
\left\langle\hat{k}_{i}\right\rangle_{\{\mathbf{x}\}} & =\left[\operatorname { m i n } _ { \{ x \} \in \{ \mathbf { x } \} } \left(\hat{k}_{i}(\{x\}), \max _{\{x\} \in\{\mathbf{x}\}}\left(\hat{k}_{i}(\{x\})\right]\right.\right. \\
\left\langle\hat{m}_{i}\right\rangle_{\{\mathbf{x}\}} & =\left[\operatorname { m i n } _ { \{ x \} \in \{ \mathbf { x } \} } \left(\hat{m}_{i}(\{x\}), \max _{\{x\} \in\{\mathbf{x}\}}\left(\hat{m}_{i}(\{x\})\right]\right.\right.
\end{aligned}
$$

This procedure does not introduce any artificial conservatism.

2. The modal envelope FRF is calculated by substituting the ranges of the modal parameters in the denominator function $\mathcal{D}_{i}(\omega)$, and subsequently inverting the resulting denominator function range. This step is performed using the interval arithmetic approach.

3. The total interval FRF is obtained by the summation of all individual modal contributions. Also this final step is performed using interval arithmetics.

Similar interval procedures can be obtained for structures with proportional or modal damping [5, 8]. 


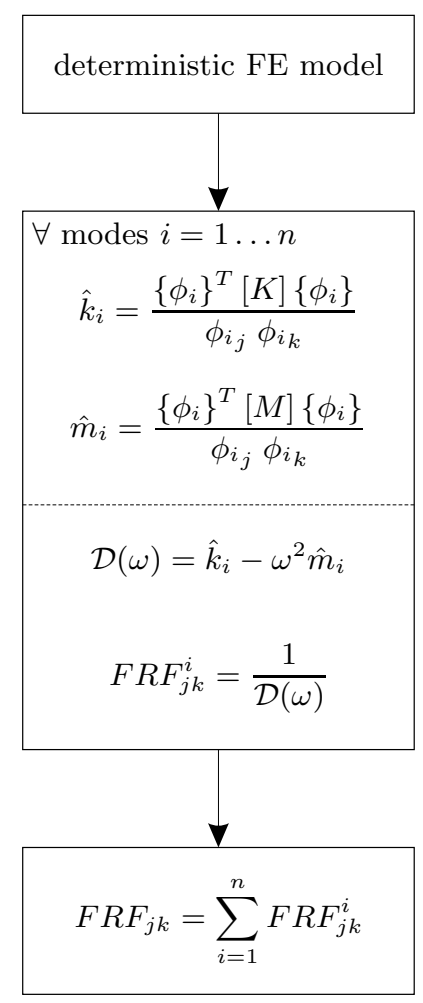

(a) Deterministic algorithm

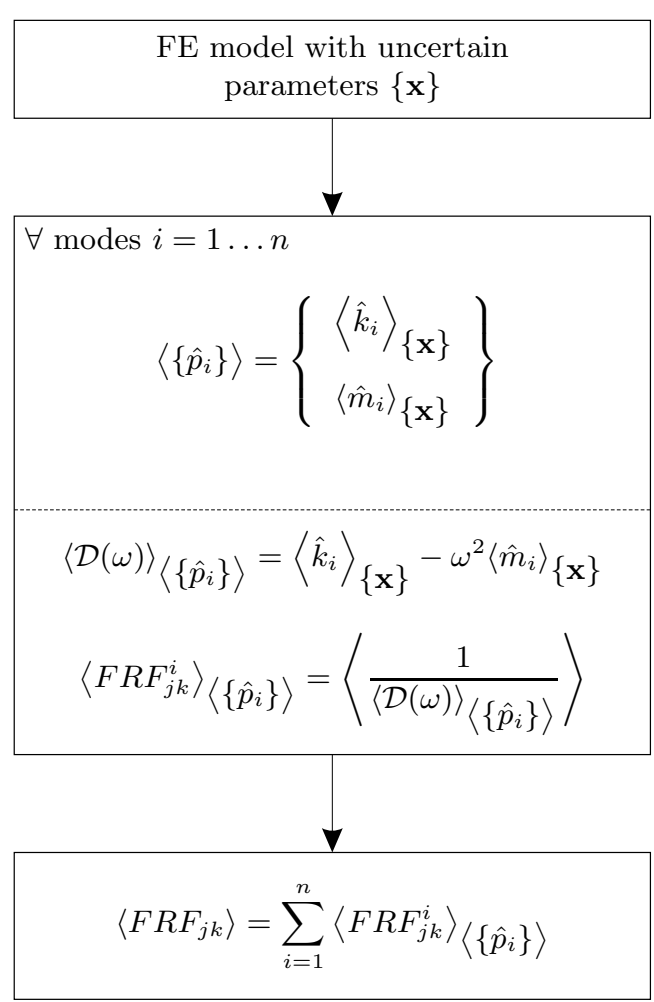

(b) Set translated algorithm

Figure 2: Translation of the deterministic modal superposition algorithm to an equivalent IFE procedure

Conservatism. In its most straightforward implementation, the modal parameter intervals $\left\langle\hat{k}_{i}\right\rangle$ and $\left\langle\hat{m}_{i}\right\rangle$ are assumed to be independent for the calculation of the modal envelope FRFs. This leads to a rectangular approximation of the (generally unknown) modal domain $\left\langle\hat{k}_{i}, \hat{m}_{i}\right\rangle$, as depicted in figure 3. Therefore, this method is referred to as the Modal Rectangle (MR) method. The figure clearly shows that this method introduces conservatism in the procedure if the actual $\left\langle\hat{k}_{i}, \hat{m}_{i}\right\rangle$ domain differs significantly from the rectangular approximation. The upper left and lower right corners represent non-physical combinations of modal mass and modal stiffness. These parameters are actually coupled through the global system matrices, and show a high degree of correlation. An enhancement of the modal domain approximation is obtained by taking the exact eigenvalue ranges $\left\langle\lambda_{i}\right\rangle$ into account, providing two lines through the origin of the graph that further delimit the $\left\langle\hat{k}_{i}, \hat{m}_{i}\right\rangle$-domain approximation, as can be seen in figure 4 . This method is referred to as the Modal Rectangle method with Eigenvalue interval correction (MRE). It has been shown that the use of the MRE domain leads to accurate FRF envelope approximations [6,9]. The eigenvalue intervals needed for the MRE approximation are calculated with an extra global minimisation and maximisation in the first step of the interval FRF procedure:

$$
\left\langle\lambda_{i}\right\rangle_{\{\mathbf{x}\}}=\left[\operatorname { m i n } _ { \{ x \} \in \{ \mathbf { x } \} } \left(\lambda_{i}(\{x\}), \max _{\{x\} \in\{\mathbf{x}\}}\left(\lambda_{i}(\{x\})\right]\right.\right.
$$

Numerical efficiency. The computational cost of an interval FRF analysis using the hybrid procedure is mainly determined by the first step of this procedure, as it requires six global optimisation procedures for each mode (Eq. (6) - (8)). For each evaluation of the objective function in the optimisation procedure, an $\mathrm{FE}$ eigenvalue analysis has to be performed, which can be very timeconsuming for large numerical models. The use of a reduced model can substantially decrease the model evaluation time, and hence increase the efficiency of the optimisation procedure drastically. 


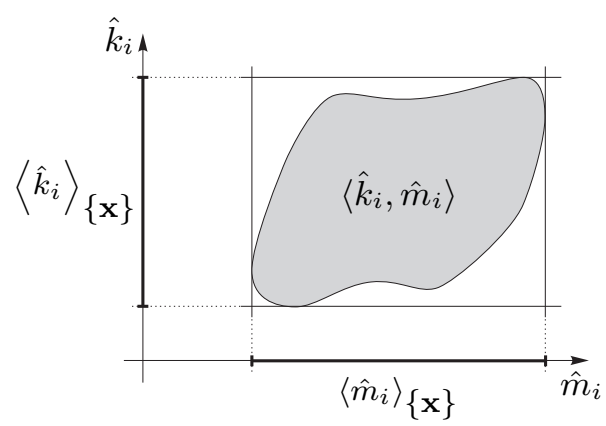

Figure 3: Graphical illustration of a mode's $\left\langle\hat{k}_{i}, \hat{m}_{i}\right\rangle$-domain, and its approximation using the modal rectangle method

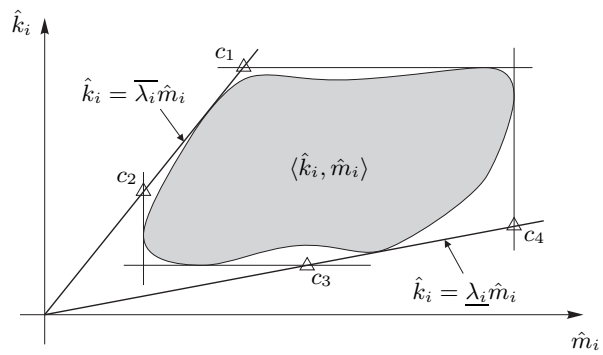

Figure 4: Effect of the introduction of the exact eigenvalue interval in the $\left\langle\hat{k}_{i}, \hat{m}_{i}\right\rangle$-domain approximation

Therefore, this paper aims at the introduction of a substructuring technique in the algorithm for interval eigenfrequency and FRF analysis.

\section{Component Mode Synthesis}

The aim of the well-known Component Mode Synthesis (CMS) method is to reduce the computational cost of large models and to enable a solution strategy in which individual components can be optimised without the need of the recalculation of the total structure. The CMS technique consists of three global steps. First the structure is divided into a residual structure and a number of components, also referred to as superelements. For each component, a set of component modes is calculated, which represent its static and dynamic behaviour. In the second step, these component modes are used to reduce the component model. In the third step, all reduced superelement models are combined with the non-reduced part of the structure to form the reduced structural system, that is further used for global FE analyses. A short overview of these basic principles and steps of the component mode synthesis method is given in this section. A complete mathematical description of the CMS technique can be found in $[10,15,16]$.

\subsection{Calculation of Component Modes}

For each component, all unconstrained degrees of freedom (dofs) are assigned to one of two specific sets. The set of boundary degrees of freedom (subscript $t$ ) contains all dofs that connect the considered component to the residual structure or to another component. The remaining dofs are assigned to the set of internal dofs (subscript o, also referred to as 'omitted' dofs). The stiffness and mass matrices of each component are assembled, and subsequently partitioned according to these sets [10]:

$$
[K]=\left[\begin{array}{ll}
\overline{K_{t t}} & K_{t o} \\
K_{o t} & K_{o o}
\end{array}\right] \quad, \quad[M]=\left[\begin{array}{ll}
\overline{M_{t t}} & M_{t o} \\
M_{o t} & M_{o o}
\end{array}\right]
$$

For each component, a set of component modes is calculated. In literature, several approaches using different types of component modes for static and dynamic reduction are described. The 
Craig-Bampton method [10] has been widely used and implemented in many finite element codes because the procedure for formulating the component modes is very straightforward, and because the method produces highly accurate results. The most commonly applied version of the CraigBampton method uses constraint modes and fixed-interface normal modes to describe the static, respectively the dynamic behaviour of a component. Each constraint mode describes the static deformation of the component when a unit displacement (or rotation) is applied to one boundary degree of freedom, while the other dofs of the t-set are restrained. The static transformation matrix $\left[G_{o t}\right]$ with all constraint modes $\left\{\phi_{i}^{C}\right\}$ as columns, has the following mathematical description:

$$
\left[G_{o t}\right]=\left[\left\{\phi_{1}^{C}\right\}\left\{\phi_{2}^{C}\right\} \ldots\left\{\phi_{t}^{C}\right\}\right]=-\left[K_{o o}\right]^{-1}\left[K_{o t}\right]
$$

The fixed-interface normal modes, which form the columns of the dynamic transformation matrix $\left[G_{o q}\right]$, are calculated from the eigenvalue analysis of the component with all boundary dofs fixed:

$$
\begin{gathered}
{\left[K_{o o}\right]\left\{\phi_{i}\right\}=\lambda_{i}\left[M_{o o}\right]\left\{\phi_{i}\right\}} \\
{\left[G_{o q}\right]=\left[\begin{array}{llll}
\left\{\phi_{1}\right\} & \left\{\phi_{2}\right\} & \cdots & \left\{\phi_{q}\right\}
\end{array}\right]}
\end{gathered}
$$

Each of the modes is assigned to a generalized degree of freedom (q-set). The accuracy of the dynamic reduction step is determined by the number of retained normal modes $q$. As the number of generalized degrees of freedom $q$ needed for an accurate description of the dynamic behaviour of the component is usually several orders smaller than the number of internal dofs, the size of the component model is drastically reduced.

\subsection{Component Reduction}

In the second step, the set of component modes is used to reduce the component stiffness and mass matrix, to produce the reduced component matrices $[10,16]$ :

$$
[K]_{\text {reduced }}=\left[\begin{array}{cc}
K_{t t} & 0 \\
0 & K_{q q}
\end{array}\right] \quad, \quad[M]_{\text {reduced }}=\left[\begin{array}{cc}
M_{t t} & M_{t q} \\
M_{q t} & M_{q q}
\end{array}\right]
$$

with:

$$
\begin{aligned}
{\left[K_{t t}\right] } & =\left[\overline{K_{t t}}\right]+\left[K_{t o}\right]\left[G_{o t}\right] \\
{\left[K_{q q}\right] } & =\left[G_{o q}\right]^{T}\left[K_{o o}\right]\left[G_{o q}\right] \\
{\left[M_{t t}\right] } & =\left[\overline{M_{t t}}\right]+\left[M_{t o}\right]\left[G_{o t}\right]+\left[G_{o t}\right]^{T}\left[M_{t o}\right]^{T}+\left[G_{o t}\right]^{T}\left[M_{o o}\right]\left[G_{o t}\right] \\
{\left[M_{t q}\right] } & =\left[M_{t o}\right]\left[G_{o q}\right]+\left[G_{o t}\right]^{T}\left[M_{o o}\right]\left[G_{o q}\right] \\
{\left[M_{q t}\right] } & =\left[M_{t q}\right]^{T} \\
{\left[M_{q q}\right] } & =\left[G_{o q}\right]^{T}\left[M_{o o}\right]\left[G_{o q}\right]
\end{aligned}
$$

These reduced matrices represent the properties of the superelement as seen at its interface with adjacent structures. In case of mass normalised normal modes, the matrices $\left[K_{q q}\right]$ and $\left[M_{q q}\right]$ simplify to respectively a diagonal matrix with the eigenvalues and a unity matrix:

$$
\left[K_{q q}\right]=\left[\Lambda_{q q}\right] \quad, \quad\left[M_{q q}\right]=\left[I_{q q}\right]
$$

\subsection{Assembly of Reduced Structural System}

In a last step, the reduced stiffness and mass matrices of all components are assembled with the non-reduced residual structure, to form the reduced stiffness and reduced mass matrix of the complete structural system. These can then be used to perform finite element analyses (eg. an eigenfrequency or frequency response function analysis) on the global structure. Data recovery for each superelement is performed by expanding the solution at the attachment points, using the same transformation matrices that were used to perform the original reduction on the superelement. 


\section{Uncertainty in an FE model with Superelements}

For the calculation of the eigenvalue intervals and the interval ranges on the modal stiffness and modal mass parameters of an uncertain structure with the global optimisation strategy, multiple eigenvalue problems have to be solved (cf. section 2). Hence, the interval finite element method for dynamic analysis (eigenvalues/FRFs) becomes very time-consuming for large systems. In this paper, the IFEM is combined with the component mode synthesis method, in order to reduce the calculation time. The uncertain parameters affecting the structural behaviour of a substructured finite element model can be located either in the non-reduced part of the structure, or in one or more superelements. Sections 4.1 and 4.2 describe the combination of the interval FE analysis with the substructuring technique for these two uncertainty location cases.

\subsection{Uncertainties in the Residual Structure}

In case that all uncertain parameters are included in the residual structure only, all components can be reduced deterministically, as described in equations (14)-(19). Hence, only the residual structure needs to be recalculated during an interval or fuzzy analysis of the global structure, while the reduced component matrices remain unchanged. In this case, the use of a substructuring technique does not change the implementation of the interval or fuzzy analysis. The numerical example given in section 5 shows that the CMS technique enhances the efficiency of the interval eigenvalue and frequency response function analysis, without compromising the accuracy of the interval results. However, this approach limits the numerical reduction and hence the gain in calculation time that can be achieved, eg. when large parts of the structure are affected by global uncertainties.

\subsection{Uncertainties on the Superelement Level}

Uncertainties in a superelement of a reduced model affect each step of the reduction procedure: the uncertainties generally affect the constraint modes, the normal modes and corresponding eigenfrequencies, the static and dynamic transformation matrices, and the reduced stiffness and mass matrices. The flow chart of figure 5 gives an overview of the global optimisation procedure for the calculation of the $k^{\text {th }}$ eigenfrequency interval of the reduced model. For the global optimisation of modal stiffness and modal mass parameters in the first step of the hybrid interval FRF procedure, a similar flow chart can be composed. In figure 5, the dashed rectangle denotes the reduction of the superelements that are affected by uncertain parameters. This paper proposes three approaches for the implementation of this reduction step: repeated component reduction, approximative component reduction based on component eigenvalue ranges, and first order Taylor series expansion of the transformation matrices. Figure 6 clarifies these three approaches to handle uncertainty on the superelement level. A legend with the notations used in figures 5 and 6 is given in table 1 .

Repeated component reduction. A first possible approach to handle uncertainties on the superelement level during an interval eigenfrequency or FRF analysis, is to recalculate the component modes, static and dynamic transformation matrices, and reduced matrices of each superelement affected by uncertain parameters, during each evaluation of the goal function of the global optimisation procedure. As in each iteration step all superelements are correctly reduced, this approach gives the correct modal parameter intervals and can hence be used as reference for approximative methods. Although the implementation of this approach is straightforward, it limits the efficiency of the substructuring technique as for each eigenvalue analysis of the global structure, one or more superelement reduction steps have to be repeated.

Approximative component reduction based on component eigenvalue ranges. Another approach to handle uncertainties on the superelement level, is to approximate the description of an uncertain superelement, such that it can represent the effect of the uncertain parameters on the static and dynamic behaviour adequately, and no component recalculation has to be performed during a global interval or fuzzy analysis of the total reduced structural system.

In literature, the approximation of uncertain superelements is most often carried out by assuming that only the eigenvalues of the superelements are affected by the uncertain parameters, and that 


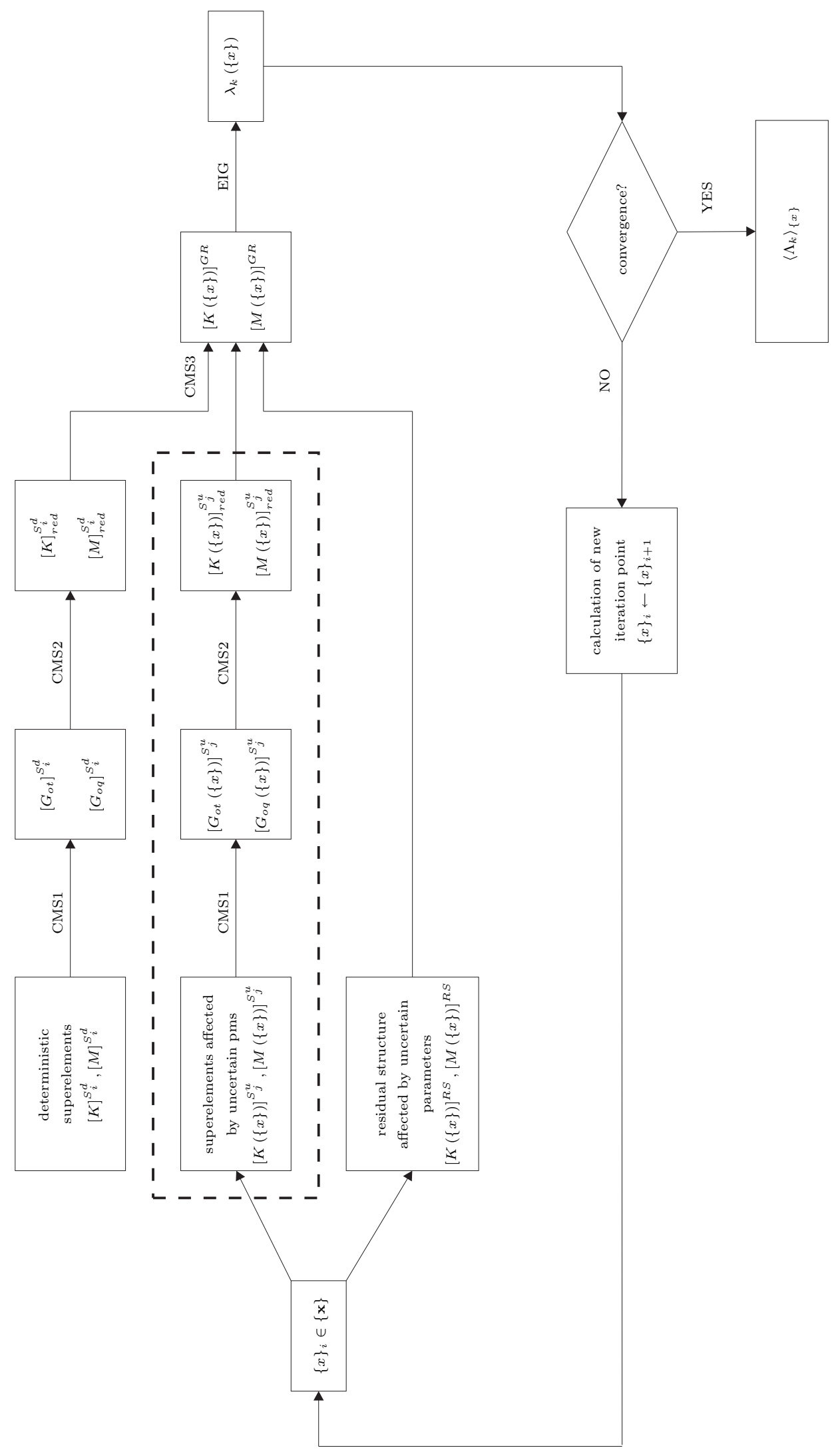

Figure 5: Flow chart for the global optimisation procedure for interval eigenfrequency analysis, in case of uncertainties on the superelement level 
repeated component reduction

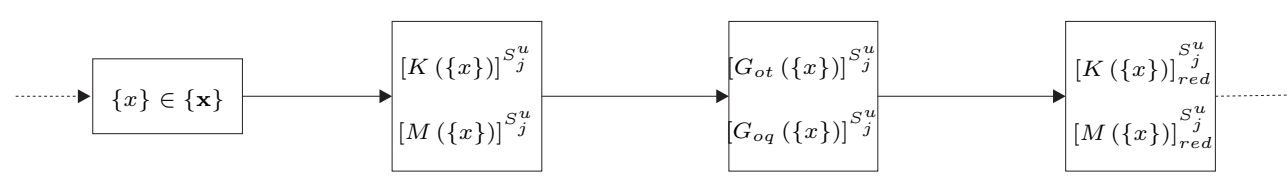

approximative component reduction based on component eigenvalue ranges

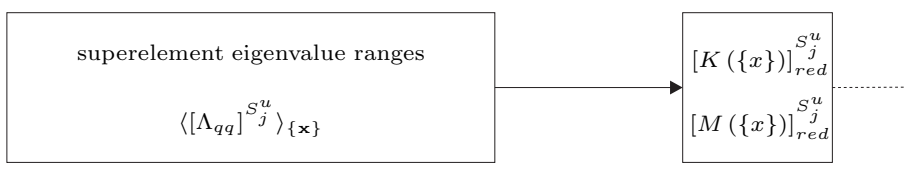

first order Taylor series expansion of transformation matrices

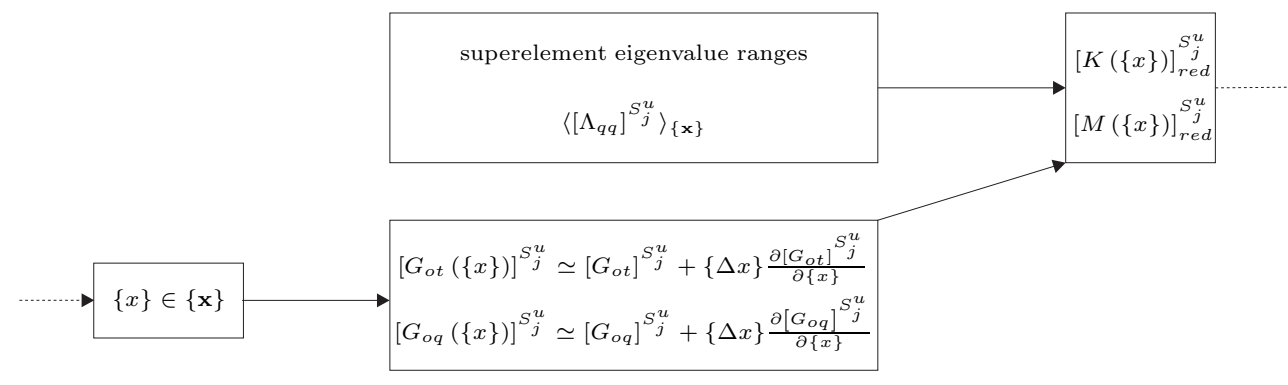

Figure 6: Three approaches for the handling of uncertainties on the superelement level

Table 1: Notations used in figures 5 and 6



the component modes remain unaffected [17, 18, 19]. For the Craig-Bampton method with mass normalized modes, this means that only the reduced matrix $\left[K_{q q}\right]$ is affected, while all other reduced matrices remain constant, as can be seen in equations (14)-(20). Therefore, in the reduction step of the algorithm, a separate interval eigenfrequency analysis is performed on the superelement in 
order to calculate the superelement eigenvalue ranges:

$$
\left\langle\left[\Lambda_{q q}\right]\right\rangle_{\{\mathbf{x}\}}=\left[\min _{\{x\} \in\{\mathbf{x}\}}\left[\Lambda_{q q}(\{x\})\right], \max _{\{x\} \in\{\mathbf{x}\}}\left[\Lambda_{q q}(\{x\})\right]\right]
$$

After this preliminary interval eigenvalue analysis on the uncertain superelement, the reduced component matrix $\left[K_{q q}(\{x\})\right]$, containing the uncertainties through the calculated eigenvalue ranges, is included in the residual structure. Hence, the uncertainty originally defined on the superelement is translated towards uncertainty affecting directly the residual structure, and no further superelement calculations are required during all global interval analyses performed on the total reduced structure, resulting in a decrease of calculation time for these global uncertainty analyses.

Taylor series expansion of the transformation matrices. The assumption that component modes such as constraint and normal modes are hardly affected by uncertain parameters might be too severe for general cases, e.g. when local geometrical uncertainties affect the component. Therefore, the method using only the eigenvalue ranges of the uncertain superelement is extended to a method that takes also the influence of superelement uncertainties on the component modes into account. To include also the component modes into an approximative reduction of an uncertain superelement, a concept for the description of uncertain vectors is required.

In this paper, a method is presented to approximate the influence of uncertain parameters on component modes of a superelement using a first order Taylor series expansion of the transformation matrices, as written here for one uncertain parameter $s$ :

$$
\begin{aligned}
{\left[G_{o t}\right]_{s_{x}} } & =\left[G_{o t}\right]_{s_{d}}+\left.\left(s_{x}-s_{d}\right) \frac{\partial\left[G_{o t}\right]}{\partial s}\right|_{s_{d}} \\
{\left[G_{o q}\right]_{s_{x}} } & =\left[G_{o q}\right]_{s_{d}}+\left.\left(s_{x}-s_{d}\right) \frac{\partial\left[G_{o q}\right]}{\partial s}\right|_{s_{d}}
\end{aligned}
$$

with $s_{x}$ and $s_{d}$ respectively the value of the uncertain parameter in the design point, and in the deterministic case. In these equations, the factor $\left(s_{x}-s_{d}\right)$ explicitly describes the influence of the uncertain parameter on the transformation matrices, while the deterministic transformation matrices and their respective derivatives preserve the correlation between the degrees of freedom.

As the static transformation matrix has an explicit expression (eq. 10), its first derivative is calculated as follows:

$$
\frac{\partial\left[G_{o t}\right]}{\partial s}=-\left[K_{o o}\right]^{-1}\left(\frac{\partial\left[K_{o t}\right]}{\partial s}+\frac{\partial\left[K_{o o}\right]}{\partial s}\left[G_{o t}\right]\right)
$$

The normal modes are calculated with the implicit expression (11). For each normal mode, the first order derivative can be calculated as proposed by Fox and Kapoor [20]:

$$
\left[\begin{array}{cc}
{\left[K_{o o}\right]-\lambda_{i}\left[M_{o o}\right]} & -\left[M_{o o}\right]\left\{\phi_{i}\right\} \\
-\left\{\phi_{i}\right\}^{T}\left[M_{o o}\right] & 0
\end{array}\right]\left\{\begin{array}{c}
\frac{\partial\left\{\phi_{i}\right\}}{\partial s} \\
\frac{\partial \lambda_{i}}{\partial s}
\end{array}\right\}=\left\{\begin{array}{c}
-\left(\frac{\partial\left[K_{o o}\right]}{\partial s}-\lambda_{i} \frac{\partial\left[M_{o o}\right]}{\partial s}\right)\left\{\phi_{i}\right\} \\
\frac{1}{2}\left\{\phi_{i}\right\}^{T} \frac{\partial\left[M_{o o}\right]}{\partial s}\left\{\phi_{i}\right\}
\end{array}\right\}
$$

For the calculation of the first order derivatives of the transformation matrices, the first derivatives of the component stiffness and mass matrices are required, as can be seen in equations (24) and (25). These can be calculated analytically, based on the finite element description of the component, or numerically, eg. with a finite difference approach.

Main advantage of this method is that no superelement recalculation (normal modes analysis/stiffness matrix inverse) has to be performed during a global uncertainty analysis of the total reduced system, as just an approximation of the transformation matrices of the uncertain superelement is used. During a global optimisation, a new iteration point in the design space is directly introduced in equations (22) and (23). In each iteration point, the superelement transformation matrices are obtained at a very low cost, once their respective sensitivities are known.

However, as only a first order approximation of the component modes is used, the method does not guarantee to reach the exact interval or fuzzy eigenfrequency results. Higher order derivatives of the component modes could lead to more accurate results for largely non-monotonic uncertain 
parameters and/or large uncertainty intervals. Also note that only the influence of uncertain parameters on the transformation matrices $\left[G_{o t}\right]$ and $\left[G_{o q}\right]$ is taken into account, thereby neglecting the influence on the component stiffness and mass matrices (cf. equations (14)-(19)). In future research, the possibilities of taking the Taylor series expansions directly on the reduced component matrices $[K]_{\text {reduced }}$ and $[M]_{\text {reduced }}$ will be investigated.

\subsection{Computational Efficiency}

The computational efficiency of the methods described in sections 4.1 and 4.2 depends strongly on the substructured FE model, the type of the uncertainties and the uncertainty locations, as each of the methods has its specific advantages and disadvantages. An analysis engineer can make a choice based on considerations regarding the size of the residual structure, the number of uncertain superelements, the type of the uncertainties (global vs. local), and the size of the uncertainty intervals.

The case in which all uncertain parameters are located in the residual structure only, has as advantage that no superelement recalculations have to be performed. This can be very interesting and efficient as for each superelement reduction a stiffness matrix inverse and a normal modes analysis have to be performed in order to determine the static respectively the dynamic transformation matrix (cf. equations (10)-(12)), which can be time-consuming for large superelements. Another advantage is that the use of a substructured FE model does not influence the implementation of the interval and fuzzy finite element method. On the other hand, in case of uncertainties affecting large parts of the model, the residual structure contains an unrealistically large number of degrees of freedom, such that the reduced FE model is almost not workable for interval or fuzzy analyses. Therefore, this method is only useful for superelement FE models with local uncertainties, eg. joints with uncertain properties.

In the case in which uncertainties are allowed on the superelement level, the uncertainties do not limit the numerical reduction that can be achieved. On the other hand, the uncertainties affecting a superelement have to be translated towards the reduced component matrices. The first approach, the repeated component reduction, is the most straightforward and the most accurate method, but has as drawback that it can be time-consuming in case of large uncertain superelements, as in each goal function evaluation of a global optimisation on the total reduced system, a matrix inverse and a normal modes analysis on the particular superelement have to be performed to recalculate the transformation matrices. Approaches 2 and 3 are numerically more efficient, but only approximate the interval results of the global reduced system. The approximative component reduction based on component eigenvalue ranges is especially suitable for global superelement uncertainties and/or small uncertainty intervals, as only the influence of the uncertainties on the component eigenvalues is taken into account, thereby neglecting the influence on the component modes. This method requires the calculation of the eigenvalue ranges of the uncertain superelement(s). The third approach, with a Taylor series expansion of the component transformation matrices, can handle the influence of uncertainties on component modes to a certain extent, hence this method is more suitable for large uncertainty intervals or local uncertainties. However, this approach is computational more expensive than the second approach as the derivatives of the component modes have to be calculated.

\section{Case Study: the GARTEUR Benchmark Problem}

\subsection{Problem Description}

The Garteur benchmark problem [21] consists of a small-scale, simplified aluminium aircraft model with a length of $1.5 \mathrm{~m}$, a wing span of $2 \mathrm{~m}$ and a mass of $44 \mathrm{~kg}$. The fuselage of the aircraft consists of a rectangular plate with a thickness of $50 \mathrm{~mm}$. The tail of $10 \mathrm{~mm}$ thickness is connected rigidly to the fuselage. The wings are connected to the fuselage through an intermediate steel plate. Wingtips are connected rigidly at both ends of the wings. Both the wings and wingtips are rectangular plates with a thickness of $10 \mathrm{~mm}$. The FE model, as illustrated in figure 7(a), contains almost 20000 degrees of freedom.

The Garteur aircraft model contains some inherent uncertainties, due to a lack of knowledge 


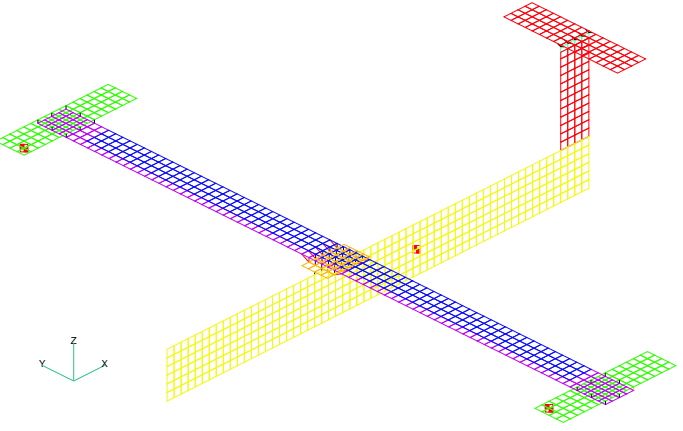

(a) Global finite element model

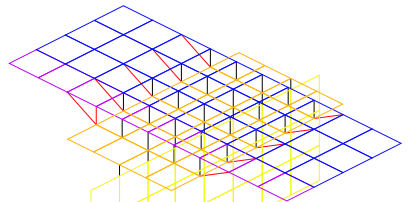

(b) Detail of the connection between fuselage and wings

Figure 7: Finite element model of the Garteur benchmark aircraft

on the physical model as well as due to uncertainty on the modelling level. Three uncertainties are considered during the dynamic analysis of the structure.

1. A first source of uncertainty is the thickness of the visco-elastic layer, glued onto a part of the wings. The uncertainty on this thickness ranges between 0.1 and $1.6 \mathrm{~mm}$, with a nominal value of $1.1 \mathrm{~mm}$.

2. A second source of uncertainty is the stiffness of a part of the connection between wings and fuselage - an inherent modelling uncertainty. In the assembled model, this connection is modelled with an interconnecting plate parallel to the wings. The fuselage is connected rigidly to this plate. The degrees of freedom of the interconnecting plate are connected rigidly to the wings, except for the DOFs on the edge of the plate. These DOFs are connected to the corresponding DOFs on the wings using linear springs. The dimension and the stiffness of the connection between the wings and the fuselage can then be varied in a continuous way by changing the stiffness of these springs. In the performed analyses this stiffness ranges between $10 \mathrm{~N} / \mathrm{m}$ and $10^{15} \mathrm{~N} / \mathrm{m}$ with a nominal value of $10^{8} \mathrm{~N} / \mathrm{m}$.

3. A third uncertainty is introduced on the Young's modulus of the wing material, with a range of $67.5 \mathrm{GPa}$ up to $68.5 \mathrm{GPa}$, with nominal value $68.0 \mathrm{GPa}$.

Two reduced models of the Garteur aircraft have been assembled. In the first model (reduced model A), four parts of the structure are modelled as deterministic superelements: the tail, fuselage and both wingtips. Hence, all uncertain parameters affect only the non-reduced residual structure. The reduced model contains 7380 degrees of freedom (versus 19898 dofs in the full model), which gives a reduction of computation time of $20 \%$. This reduction affects every goal function evaluation in the global optimisation of the aircraft eigenfrequencies and modal stiffness and modal mass parameters; therefore, the same proportional time gain is achieved for the total IFEM and FFEM procedure. By substructuring the Garteur aircraft such that all model parts affected by uncertain parameters are located in the residual structure only, the number of degrees of freedom of this global reduced system remains quite large. In this case, the strength of the component mode synthesis method is not fully exploited, as a model reduction normally leads to a model size of 500 à 1000 dofs. However, this reduced model has as advantage that no superelements are affected by uncertain parameters, hence no superelement recalculations have to be performed during interval or fuzzy analyses. In the second reduced model, further denoted as reduced model B, also the wings of the aircraft are modelled as a separate superelement, leaving only the interconnecting steel plate as non-reduced part. This assembled model, containing the residual structure and the reduced models of fuselage-tail and wings-wingtips, only contains 628 degrees of freedom. Hence, the computation time is further reduced drastically. Table 2 gives an overview of the different Garteur aircraft finite element models, with indication of the number of degrees of freedom in 
the residual structure and the superelements. The terminology and notation used in this table are compatible with the FE software MSC.Nastran [16]. For each of the models, the size of the residual structure is denoted, with the degrees of freedom subdivided into degrees of freedom that are constrained during the analyses, and degrees of freedom that are free to displace/rotate. For both reduced Garteur FE models, the size of the deterministic and uncertain superelements is indicated, before reduction as well as after reduction. Before reduction, the degrees of freedom are subdivided between boundary and internal degrees of freedom. After reduction, the dofs are subdivided according to the t-set (boundary dofs, connected to the constraint modes) and the q-set (generalized dofs, connected to the component normal modes).

Table 3 gives a comparison of the first 14 eigenfrequencies of the full (i.e. non-reduced) model versus the two superelement models, as well as the relative differences per thousand. For both reduced models, the deterministic eigenfrequencies are accurately predicted. Figure 8 shows the mode shapes of the first 14 modes. Modes 1, 2, 6, 9, 13 and 14 are the first six bending modes of the wings. The first, third and fifth bending modes are symmetric, with both wings vibrating in phase, while the remainder of the structure hardly moves. The second, fourth and sixth bending modes are antisymmetric, with both wings vibrating in anti-phase. In these modes, the fuselage and tail tilt around the x-axis. Modes 3 and 4 are torsion modes (anti-phase, respectively in phase) of the wings around the y-direction, while the remainder of the structure stands still. Modes 7 and 8 are bending modes of the wings in the xy-plane, with the wings in anti-phase in mode 7 and in phase in mode 8 . Modes 5 and 12 are modes that combine different movements: bending and torsion of the wings, and tilting of the fuselage and tail. Mode 10 is a local torsion of the tail wrt. the fuselage. Mode 11 is the first bending of the fuselage.

Table 2: Overview of the different Garteur aircraft FE models and their degrees of freedom

\begin{tabular}{|c|c|c|c|}
\hline & full model & reduced model $\mathrm{A}$ & reduced model B \\
\hline $\begin{array}{l}\text { number of dofs } \\
\text { in the residual } \\
\text { structure }\end{array}$ & $\begin{array}{l}19896 \text { dofs } \\
\text { - constrained: } 3542 \\
\text { - free: } 16354\end{array}$ & $\begin{array}{l}7391 \text { dofs } \\
\text { - constrained: } 1295 \\
\text { - free: } 6096\end{array}$ & $\begin{array}{l}628 \text { dofs } \\
\text { - constrained: } 86 \\
\text { - free: } 542\end{array}$ \\
\hline $\begin{array}{l}\text { uncertain } \\
\text { superelements }\end{array}$ & / & $\begin{array}{l}\text { Su } \\
- \\
- \\
-\end{array}$ & $\begin{array}{l}\text { before reduction: } 6948 \\
\text { boundary: } 150 \\
\text { internal: } 6798 \\
\text { after reduction: } 185 \\
\text { t-set: } 150 \\
\text { q-set: } 35\end{array}$ \\
\hline $\begin{array}{l}\text { deterministic } \\
\text { superelements }\end{array}$ & / & $\begin{array}{l}\text { superelement TAIL } \\
\text { - before reduction: } 2544 \\
\text { boundary: } 54 \\
\text { internal: } 2490 \\
\text { superelement LEFT WING } \\
\text { - before reduction: } 1446 \\
\text { boundary: } 48 \\
\text { internal: } 1398 \\
\text { superelement RIGHT WIN } \\
\text { - before reduction: } 1446 \\
\text { boundary: } 48 \\
\text { internal: } 1398 \\
\text { superelement FUSELAGE } \\
\text { - before reduction: } 7344 \\
\text { boundary: } 42 \\
\text { internal: } 7302\end{array}$ & $\begin{array}{l}\text { - after reduction: } 78 \\
\text { t-set: } 54 \\
\text { q-set: } 24 \\
\text { GTIP } \\
\text { - after reduction: } 60 \\
\text { t-set: } 48 \\
\text { q-set: } 12 \\
\text { NGTIP } \\
\text { - after reduction: } 60 \\
\text { t-set: } 48 \\
\text { q-set: } 12 \\
\\
\text { - after reduction: } 77 \\
\text { t-set: } 42 \\
\text { q-set: } 35\end{array}$ \\
\hline
\end{tabular}




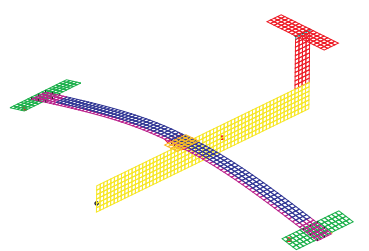

(a) mode 1

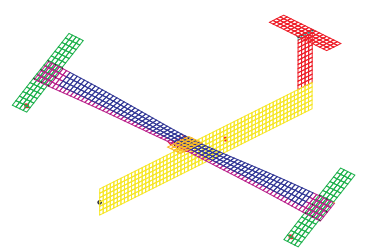

(d) mode 4



(g) mode 7

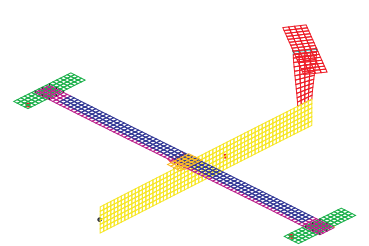

(j) mode 10

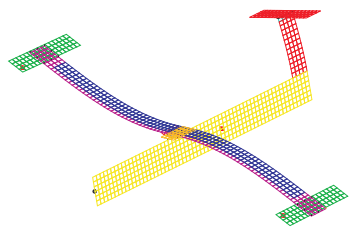

(b) mode 2

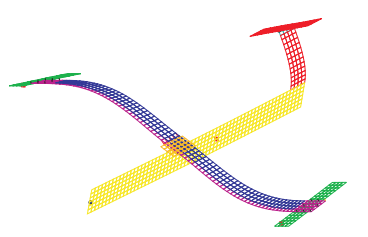

(e) mode 5

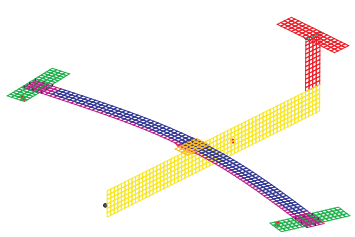

(h) mode 8

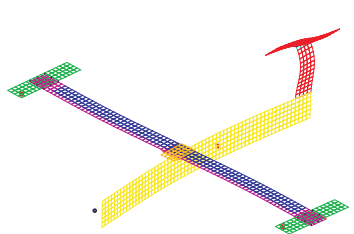

(k) mode 11

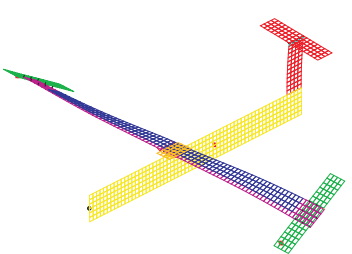

(c) mode 3

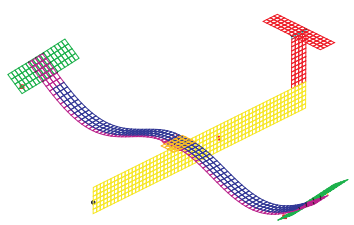

(f) mode 6

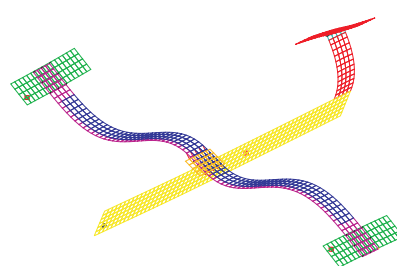

(i) mode 9

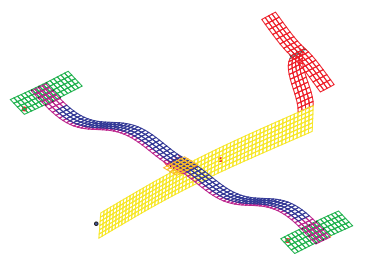

(1) mode 12

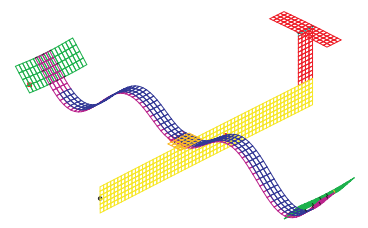

(m) mode 13

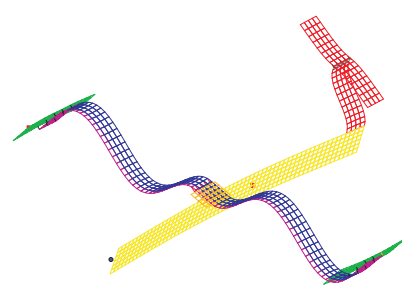

(n) mode 14

Figure 8: Mode shapes of the first 14 modes of the Garteur benchmark aircraft 
Table 3: Eigenfrequency values $[\mathrm{Hz}]$ for the non-reduced model and both superelement models, for the deterministic case

\begin{tabular}{cccccc}
\hline $\begin{array}{c}\text { mode } \\
\text { no. }\end{array}$ & $\begin{array}{c}\text { full } \\
\text { model }\end{array}$ & $\begin{array}{c}\text { reduced } \\
\text { model A }\end{array}$ & $\begin{array}{c}\text { difference } \\
(\%)\end{array}$ & $\begin{array}{c}\text { reduced } \\
\text { model B }\end{array}$ & $\begin{array}{c}\text { difference } \\
(\%)\end{array}$ \\
\hline 1 & 5.8095 & 5.8095 & 0.00 & 5.8093 & -0.02 \\
2 & 15.203 & 15.203 & 0.00 & 15.203 & -0.03 \\
3 & 33.077 & 33.102 & 0.76 & 33.102 & 0.74 \\
4 & 33.204 & 33.232 & 0.84 & 33.233 & 0.89 \\
5 & 35.609 & 35.614 & 0.14 & 35.614 & 0.14 \\
6 & 46.641 & 46.654 & 0.30 & 46.653 & 0.27 \\
7 & 49.821 & 49.820 & -0.02 & 49.826 & 0.09 \\
8 & 54.029 & 54.025 & -0.07 & 54.054 & 0.46 \\
9 & 62.358 & 62.387 & 0.47 & 62.385 & 0.43 \\
10 & 67.595 & 67.595 & 0.00 & 67.595 & 0.00 \\
11 & 100.24 & 100.24 & 0.00 & 100.24 & 0.05 \\
12 & 128.81 & 128.89 & 0.62 & 128.89 & 0.59 \\
13 & 137.44 & 137.77 & 2.40 & 137.77 & 2.34 \\
14 & 150.85 & 151.04 & 1.26 & 151.04 & 1.24 \\
\hline
\end{tabular}

\subsection{Uncertainties in the Residual Structure}

Interval eigenfrequency analysis. An interval eigenfrequency analysis with the three above mentioned uncertain parameters has been performed on the reduced model A, i.e. with all uncertain parameters in the residual structure. Table 4 gives the resulting eigenfrequency intervals of the first 14 modes for the reduced model, compared to the exact results of the full model. The results prove that the use of a substructuring technique with all uncertain parameters in the residual structure, enhances the efficiency of the IFEM without compromising the accuracy of the interval results. Comparing the interval eigenfrequency results with the mode shapes described in section 5.1, it is clear that the uncertain parameters affect mainly the six bending modes of the wings. The widest eigenfrequency intervals are detected on the symmetric bending modes (modes 1, 6 and 13), followed by the eigenfrequency intervals on the antisymmetric bending modes (modes 2, 9 and 14). Also the torsion modes 3 and 4 are clearly affected. On the other hand, the local modes on the tail and the fuselage (modes 10 and 11) are hardly affected, which follows directly from the fact that all uncertain parameters are located on the wings of the aircraft.

During the analyses, it was noticed that the uncertainty on the thickness of the visco-elastic layer has a non-monotonic influence on the eigenfrequencies. Consequently, the global optimisation strategy was required to find the correct extrema on the eigenfrequencies.

Interval FRF analysis. The influence of the three uncertain parameters on the proportionally damped interval FRF between the wingtips of the aircraft model is investigated. The input and output dofs are indicated in figure $7(\mathrm{a})$. For the calculation of the interval FRF, 14 modes are taken into account, covering a frequency range up to $160 \mathrm{~Hz}$. The damping ratios of all considered modes have values between $1.0 \%$ and $2.8 \%$. Figure 9 presents the results of the interval FRF analysis compared with the results of a Monte Carlo Simulation with 100 samples. The upper and lower FRF bound calculated with the IFEM gives a narrow envelope of the Monte Carlo results for the entire frequency domain. The use of the CMS method induces hardly any loss of accuracy as the interval FRF results of the full and the reduced model coincide well.

\subsection{Uncertainties on the Superelement Level}

An interval and fuzzy eigenfrequency analysis have been performed on the reduced model B, with only the Young's modulus of the wing material as uncertain parameter, which is affecting only the wing superelement. To have a clearer view on the effect of an uncertainty on the superelement level on the dynamic behaviour of the global structure, the input uncertainty interval on the Young's 
Table 4: Eigenfrequency intervals $[\mathrm{Hz}]$ and width of the intervals (\%) of the first 14 modes of the Garteur aircraft model, for the full model, the reduced model A and the reduced model B (repeated component reduction)

\begin{tabular}{ccccccc}
\hline & \multicolumn{2}{c}{ full model } & \multicolumn{2}{c}{ reduced model A } & \multicolumn{2}{c}{ reduced model B } \\
\hline $\begin{array}{c}\text { mode } \\
\text { no. }\end{array}$ & $\begin{array}{c}\text { frequency } \\
\text { interval }\end{array}$ & $\begin{array}{c}\text { interval } \\
\text { width }(\%)\end{array}$ & $\begin{array}{c}\text { frequency } \\
\text { interval }\end{array}$ & $\begin{array}{c}\text { interval } \\
\text { width }(\%)\end{array}$ & $\begin{array}{c}\text { frequency } \\
\text { interval }\end{array}$ & $\begin{array}{c}\text { interval } \\
\text { width }(\%)\end{array}$ \\
\hline 1 & $5.4886-5.9137$ & 7.32 & $5.4884-5.9137$ & 7.32 & $5.4868-5.9134$ & 7.34 \\
2 & $14.727-15.365$ & 4.20 & $14.728-15.365$ & 4.19 & $14.729-15.365$ & 4.18 \\
3 & $32.417-33.379$ & 2.91 & $32.442-33.404$ & 2.91 & $32.434-33.404$ & 2.93 \\
4 & $32.485-33.532$ & 3.15 & $32.512-33.560$ & 3.15 & $32.503-33.563$ & 3.19 \\
5 & $35.263-35.766$ & 1.41 & $35.267-35.772$ & 1.42 & $35.265-35.772$ & 1.42 \\
6 & $43.926-47.804$ & 8.31 & $43.937-47.818$ & 8.32 & $43.922-47.817$ & 8.35 \\
7 & $49.269-50.007$ & 1.48 & $49.269-50.008$ & 1.48 & $49.272-50.012$ & 1.49 \\
8 & $53.482-54.347$ & 1.60 & $53.475-54.344$ & 1.61 & $53.511-54.380$ & 1.61 \\
9 & $59.361-63.617$ & 6.83 & $59.382-63.647$ & 6.84 & $59.367-63.644$ & 6.86 \\
10 & $67.583-67.603$ & 0.03 & $67.583-67.603$ & 0.03 & $67.583-67.603$ & 0.03 \\
11 & $100.10-100.33$ & 0.23 & $100.10-100.33$ & 0.23 & $100.11-100.34$ & 0.23 \\
12 & $126.26-129.58$ & 2.58 & $126.37-129.64$ & 2.54 & $126.35-129.64$ & 2.55 \\
13 & $129.50-141.12$ & 8.45 & $129.77-141.47$ & 8.49 & $129.75-141.47$ & 8.51 \\
14 & $143.89-153.91$ & 6.64 & $143.99-154.14$ & 6.72 & $143.96-154.13$ & 6.73 \\
\hline
\end{tabular}

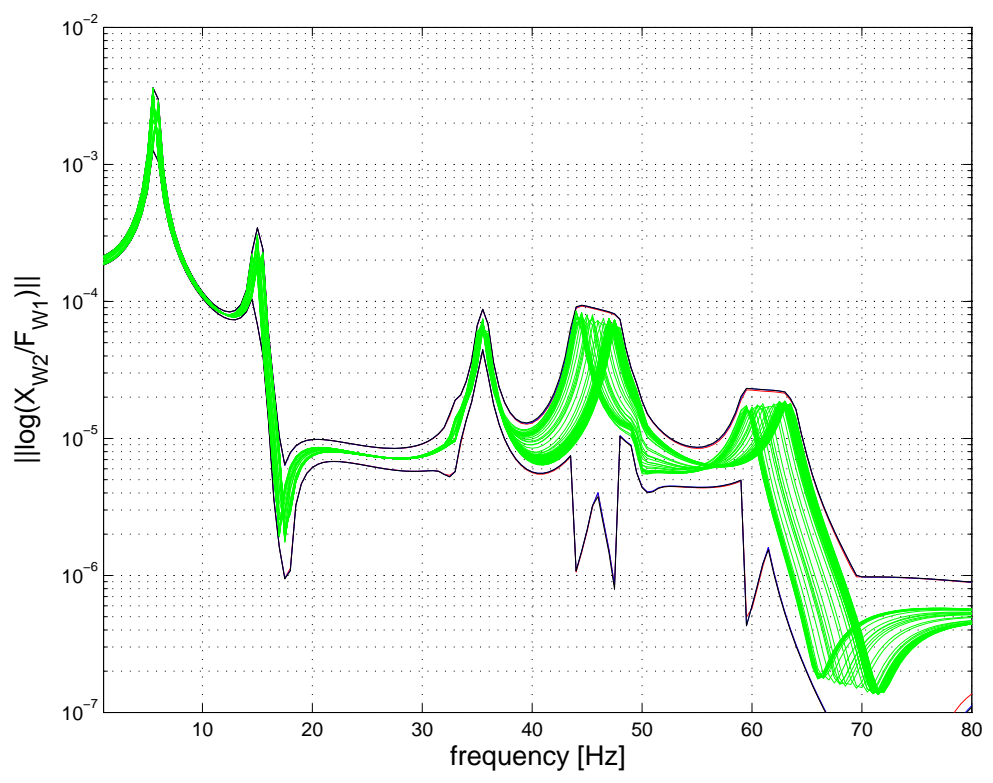

Figure 9: Amplitude of the damped interval FRF of the Garteur aircraft model, compared with 100 Monte Carlo samples

modulus of the wing material has been enlarged to [60.0 - 76.0] GPa.

On the reduced model B, the three approaches introduced in section 4.2 have been applied. Following the first approach, the uncertain superelement is recalculated for each goal function evaluation of each optimisation procedure. This approach gives the correct eigenfrequency results for the reduced model, which will be used as reference. In the two other approaches, the physical uncertainty of the Young's modulus of the wing material is transferred to the representation of the uncertain superelement by its reduced matrices. In the second approach, only the range of eigenvalues corresponding to the fixed-interface normal modes of the wing superelement are taken into account. In the third approach, also a first order approximation of the transformation matrices is used for 
the calculation of the reduced matrices of the wing superelement in each of the goal function evaluations. Table 5 lists the eigenfrequency intervals as calculated with the three approaches.

The interval results indicate that the two approximative methods lead to a small underestimation of the width of the eigenfrequency intervals. In the second approach only the influence of the uncertainty on the superelement eigenfrequencies is taken into account; yet the results are quite accurate, due to the fact that the input uncertainty is a global model parameter that mainly affects the eigenfrequencies while the shape of the component modes remains almost unaffected. The approximation of the transformation matrices by a first order Taylor series expansion leads to very similar interval results. A small improvement of the interval eigenfrequency results is noticed on all antisymmetric bending modes of the wings (modes 2, 9 and 14), on the symmetric bending mode 13 , and on the torsion modes 3 and 7 . Note that due to the enlarged uncertainty interval on the Young's modulus of the wing material, mainly the eigenfrequency intervals on the torsion modes and the bending modes in the xy-plane have increased.

In this case study, both the second and third approach of section 4.2 give accurate results for the eigenfrequency intervals of the Garteur aircraft due to an uncertainty on the Young's modulus of the wing material, while the calculation time is reduced by $28 \%$ wrt. the non-reduced model, as no superelement recalculation has to be performed during the global uncertainty analysis. For the third approach, the extra calculation time needed for the Taylor approximation of the transformation matrices can be controlled by the number of component modes taken into account during the analysis. For local input uncertainties (eg. local geometric uncertainties), the assumption that the component modes are not influenced by the uncertain parameters, can lead to larger underestimations of the correct eigenfrequency interval results. In these cases, another approach for the representation of uncertainties on the superelement level is required.

Table 5: Comparison of the interval eigenfrequency results $[\mathrm{Hz}]$ of the three approaches

\begin{tabular}{ccccccc}
\hline & \multicolumn{2}{c}{$\begin{array}{c}\text { repeated component } \\
\text { reduction }\end{array}$} & \multicolumn{2}{c}{$\begin{array}{c}\text { approximate component } \\
\text { reduction, based on } \\
\text { substr eigenvalue ranges }\end{array}$} & \multicolumn{2}{c}{$\begin{array}{c}\text { first order Taylor } \\
\text { series expansion of } \\
\text { transformation matrices }\end{array}$} \\
\hline $\begin{array}{c}\text { mode } \\
\text { no. }\end{array}$ & $\begin{array}{c}\text { frequency } \\
\text { interval }\end{array}$ & $\begin{array}{c}\text { interval } \\
\text { width }(\%)\end{array}$ & $\begin{array}{c}\text { frequency } \\
\text { interval }\end{array}$ & $\begin{array}{c}\text { interval } \\
\text { width }(\%)\end{array}$ & $\begin{array}{c}\text { frequency } \\
\text { interval }\end{array}$ & $\begin{array}{c}\text { interval } \\
\text { width (\%) }\end{array}$ \\
\hline 1 & $5.4630-6.1351$ & 11.57 & $5.4640-6.1339$ & 11.53 & $5.4704-6.1276$ & 11.31 \\
2 & $14.447-15.885$ & 9.46 & $14.453-15.880$ & 9.39 & $14.449-15.883$ & 9.43 \\
3 & $31.200-34.827$ & 10.96 & $31.204-34.821$ & 10.93 & $31.203-34.823$ & 10.94 \\
4 & $31.283-35.062$ & 11.37 & $31.286-35.059$ & 11.36 & $31.285-35.060$ & 11.36 \\
5 & $34.671-36.512$ & 5.17 & $34.683-36.500$ & 5.10 & $34.684-36.500$ & 5.10 \\
6 & $43.889-49.251$ & 11.49 & $43.898-49.240$ & 11.45 & $43.900-49.239$ & 11.45 \\
7 & $48.120-51.287$ & 6.36 & $48.165-51.239$ & 6.17 & $48.139-51.262$ & 6.27 \\
8 & $50.867-57.050$ & 11.44 & $50.927-56.975$ & 11.19 & $50.927-56.975$ & 11.19 \\
9 & $59.676-64.995$ & 8.53 & $59.683-64.987$ & 8.50 & $59.674-64.998$ & 8.53 \\
10 & $67.540-67.650$ & 0.16 & $67.542-67.648$ & 0.16 & $67.541-67.649$ & 0.16 \\
11 & $99.573-100.88$ & 1.30 & $99.592-100.85$ & 1.26 & $99.603-100.84$ & 1.23 \\
12 & $125.81-130.79$ & 3.87 & $125.83-130.76$ & 3.82 & $125.83-130.76$ & 3.83 \\
13 & $129.67-145.36$ & 11.39 & $129.70-145.32$ & 11.34 & $129.70-145.33$ & 11.35 \\
14 & $145.36-157.35$ & 7.93 & $145.36-157.33$ & 7.92 & $145.35-157.34$ & 7.94 \\
\hline
\end{tabular}

For the reduced model B, a fuzzy eigenfrequency analysis has been performed. The membership function used for the input uncertain parameter has a triangular shape, as indicated in figure 10. Figures 11 and 12 give the fuzzy results for eigenmodes 1, 7,11 and 12, as calculated with the first two approaches. The fuzzy plots give a clear indication of the influence of the width of the input uncertainty interval on the eigenfrequency intervals of the global structure. These figures also prove that in this case of a global uncertain parameter, the second method gives a good approximation of the fuzzy eigenfrequencies of the total structure. 


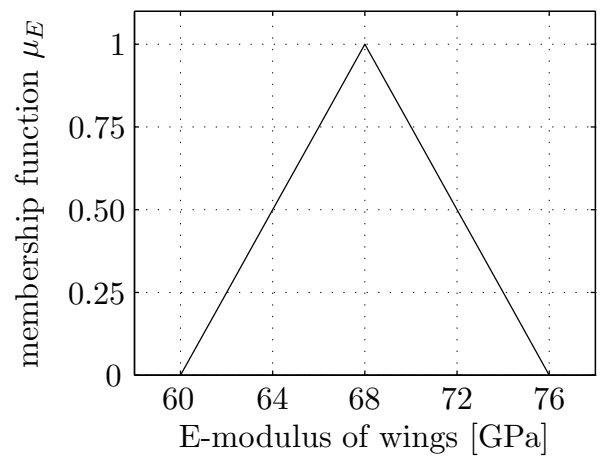

Figure 10: Triangular membership function for the Young's modulus of the wing material
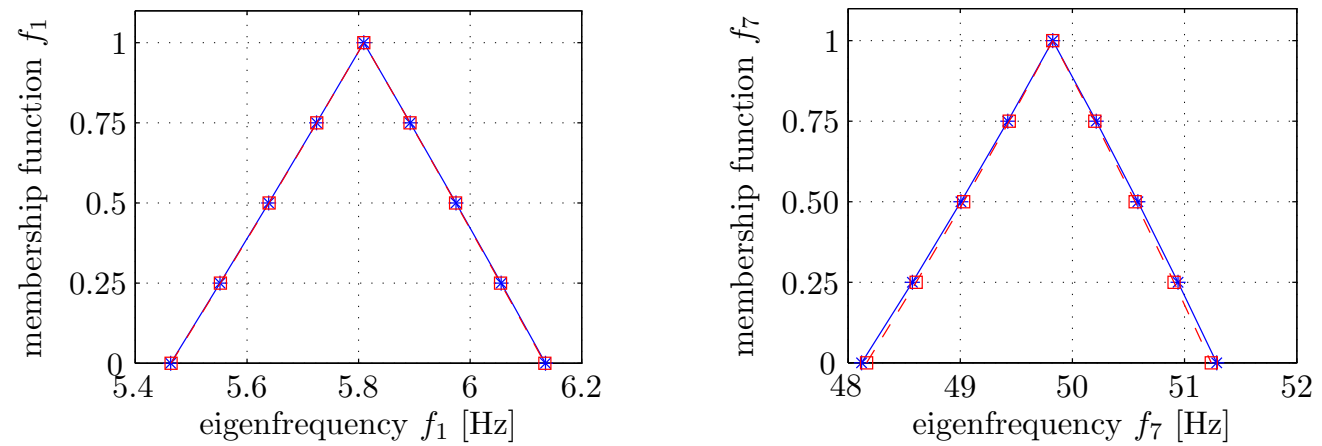

Figure 11: Membership function of the $1^{\text {st }}$ and $7^{\text {th }}$ eigenfrequency of the Garteur aircraft. Solid line: repeated component reduction, dotted line: approximative component reduction based on component eigenvalue ranges
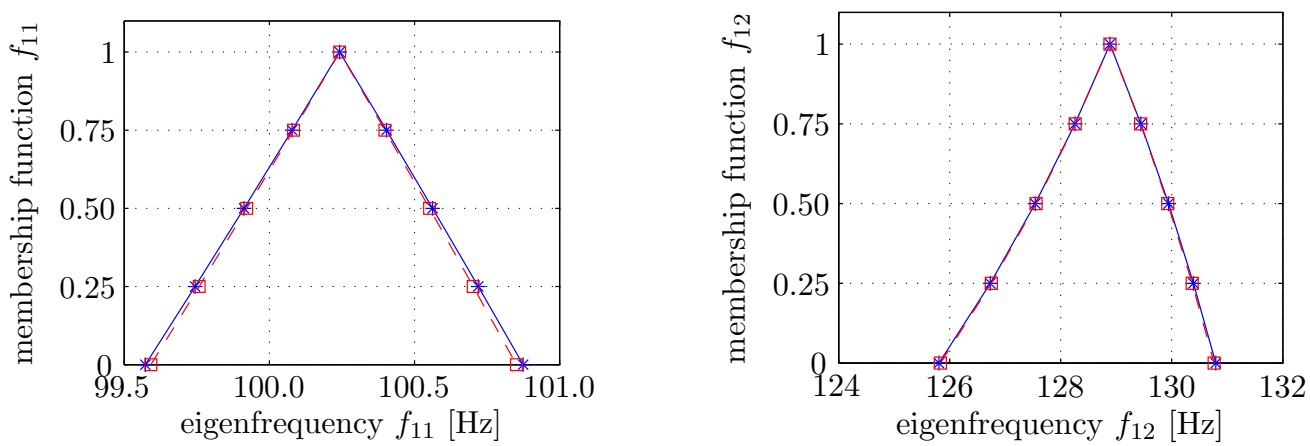

Figure 12: Membership function of the $11^{\text {th }}$ and $12^{\text {th }}$ eigenfrequency of the Garteur aircraft. Solid line: repeated component reduction, dotted line: approximative component reduction based on component eigenvalue ranges

\subsection{Computational Efficiency}

Comparing the efficiency of the reduced model A wrt. to the full (i.e. non-reduced) model for the interval eigenfrequency analysis with the three uncertain parameters of section 5.1, a time gain of $20 \%$ is achieved, hardly without any loss of accuracy. The interval analysis using the reduced model B combined with the repeated component reduction approach, gives the same results (cf. table 4). In this particular case of the Garteur aircraft with all uncertain parameters affecting the large structure of the wings, reduced model A has been detected slightly more efficient than reduced model B (in case of the repeated component reduction approach). This can be explained from the comparison of the number of degrees of freedom in both residual structures and the wings superelement. The residual structure of reduced model A contains 6096 unconstrained degrees of 
freedom, hence in each goal function evaluation during a global optimisation procedure, a normal modes analysis of a square system of 6096 dofs has to be solved. The residual structure of reduced model B contains only 524 unconstrained dofs, however due to the repeated component reduction approach, also a normal modes analysis and a stiffness matrix inverse on the wings superelement (5589 unconstrained dofs) has to be solved to recalculate the superelement transformation matrices, hence the computational advantage of the much smaller residual structure does not materialise.

The use of an approximative component reduction method in combination with reduced model B, brings in an additional calculation time gain with respect to the full model and the reduced model A. The case study clearly shows that in case of a global uncertain parameter, the approximations of the interval results are very good. In case of local uncertain parameters or large uncertainty intervals, the exta calculation time needed for the approach using the Taylor series expansion of the transformation matrices will be more justified than in the presented case with only a global uncertain parameter.

\section{Conclusion}

This paper uses the non-probabilistic interval and fuzzy finite element methods for the dynamic analysis of a structure with uncertain parameters. A reduction of the calculation time of the uncertainty analyses is obtained by the application of a substructuring technique, i.e. the component mode synthesis method. The paper first describes the basis principles of fuzzy sets and the fuzzy finite element method for eigenvalue and frequency response function analysis. Then an overview of the basic steps of the component mode synthesis technique is given. The consequences of uncertainties on the mathematical description of the substructuring technique are investigated, for uncertainties located either in the residual structure or on the superelement level. For the latter case, special attention is paid to the consequences of these uncertainties on the numerical representation of the component modes and the reduced matrices of an uncertain superelement. In this paper, the eigenvalue intervals and a first order Taylor series expansion of the component modes of an uncertain superelement are used to approximate the interval eigenfrequencies and modal stiffness and modal mass parameters of the reduced structural system. The case study of the Garteur benchmark aircraft proves that in case that all uncertain parameters are located in the residual structure, the component mode synthesis technique enhances the efficiency of the IFEM without compromising the accuracy of the interval eigenvalue results and the envelope FRFs. In case of uncertainties on the superelement level, the interval and fuzzy eigenfrequency results of the approximative approaches are promising in case of a global superelement uncertainty. In future research, the non-monotonic influence of local uncertain parameters on the approximate representation of uncertain superelements will be investigated.

\section{Acknowledgments}

H. De Gersem is research assistant of the Research Foundation - Flanders (FWO-Vlaanderen). D. Moens is postdoctoral fellow of the Research Foundation - Flanders (FWO-Vlaanderen).

\section{References}

[1] A. Haldar and S. Mahadevan. Reliability Assessment using Stochastic Finite Element Analysis. John Wiley \& Sons, Inc., New York, first edition, 2000.

[2] I. Elishakoff. Essay on uncertainties in elastic and viscoelastic structures: from A.M. Freudenthal's criticism to modern convex modelling. Computers \& Structures, 56(2):871-895, 1995.

[3] L. Zadeh. Fuzzy sets. Information \& Control, 8(3):338-353, 1965.

[4] S.S. Rao and P. Sawyer. Fuzzy finite element approach for the analysis of imprecisely defined systems. AIAA Journal, 33(12):2364-2370, 1995.

[5] D. Moens. A non-probabilistic finite element approach for structural dynamic analysis with uncertain parameters. PhD thesis, Katholieke Universiteit Leuven, Belgium, 2002. 
[6] D. Moens and D. Vandepitte. An interval finite element approach for the calculation of envelope frequency response functions. International Journal for Numerical Methods in Engineering, 61(14):2480-2507, 2004.

[7] D. Moens and D. Vandepitte. A fuzzy finite element procedure for the calculation of uncertain frequency response functions of damped structures: Part 1 - procedure. Journal of Sound and Vibration, 288(3):431-462, 2005.

[8] D. Moens and D. Vandepitte. Frequency response function analysis of structures with fuzzy modal damping parameters. In Proceedings of the 7th AIAA Non-Deterministic Approaches Forum, Austin, 2005.

[9] H. De Gersem, D. Moens, W. Desmet, and D. Vandepitte. A fuzzy finite element procedure for the calculation of uncertain frequency response functions of damped structures: Part 2 numerical case studies. Journal of Sound and Vibration, 288(3):463-486, 2005.

[10] R.R. Craig and M.C.C. Bampton. Coupling of substructures for dynamic analyses. AIAA Journal, 6(7):1313-1319, 1968.

[11] D. Moens and D. Vandepitte. Non-probabilistic approaches for non-deterministic dynamic FE analysis of imprecisely defined structures. In Proceedings of the International Conference on Noise and Vibration Engineering, ISMA 2004, pages 3095-3119, Leuven, 2004.

[12] W. Dong and H. Shah. Vertex method for computing functions of fuzzy variables. Fuzzy Sets and Systems, 24(1):65-78, 1987.

[13] M. Hanss. The transformation method for the simulation and analysis of systems with uncertain parameters. Fuzzy Sets and Systems, 130(3):277-289, 2002.

[14] S. Donders, D. Vandepitte, J. Van de Peer, and W. Desmet. Assessment of uncertainty on structural dynamic responses with the short transformation method. Journal of Sound and Vibration, 288(3):523-549, 2005.

[15] R.R. Craig Jr. A review of time-domain and frequency-domain component mode synthesis methods. Journal of Modal Analysis, 2(2):59-72, 1987.

[16] The MacNeal-Schwendler Corporation, Los Angeles. MSC.Nastran superelement user's guide, 2001.

[17] B.R. Mace and P.J. Shorter. A local modal/perturbational method for estimating frequency response statistics of built-up structures with uncertain properties. Journal of Sound and Vibration, 242(5):793-811, 2001.

[18] B. Lallemand, A. Cherki, T. Tison, and P. Level. Fuzzy modal finite element analysis of structures with imprecise material properties. Journal of Sound and Vibration, 220(2):353364, 1999.

[19] B. Van den Nieuwenhof. Stochastic finite elements for elastodynamics: random field and shape uncertainty modelling using direct and modal perturbation-based approaches. PhD thesis, Université Catholique de Louvain, Louvain-La-Neuve, Belgium, 2003.

[20] R.L. Fox and M.P. Kapoor. Rates of change of eigenvalues and eigenvectors. AIAA Journal, 6(12):2426-2429, 1968.

[21] M. Degener and M. Hermes. Ground vibration test and finite element analysis of the garteur SM-AG19 testbed. Technical Report IB 232-96 J 08, DLR - German Aerospace Research Establishment, Institute for Aeroelasticity, Oct 1996. 